Meta

Journal des traducteurs

Translators' Journal

\title{
Comment le dieu biblique peut " aller au hasard " en hébreu mais pas en traduction
}

\section{Henri Atlan}

Volume 40, numéro 3, septembre 1995

La traduction, qu'est-ce à dire? Phénoménologies de la traduction

URI : https://id.erudit.org/iderudit/001916ar

DOI : https://doi.org/10.7202/001916ar

Aller au sommaire du numéro

Éditeur(s)

Les Presses de l'Université de Montréal

ISSN

0026-0452 (imprimé)

1492-1421 (numérique)

Découvrir la revue

Citer cet article

Atlan, H. (1995). Comment le dieu biblique peut « aller au hasard » en hébreu mais pas en traduction. Meta, 40(3), 508-513. https://doi.org/10.7202/001916ar
Résumé de l'article

Medical doctor, biologist and professor in Paris and Jerusalem, the author has developed theories on the complexity and the self-organization of living organisms (Entre le cristal et la fumée, Seuil. 1979). Drawing on his profound knowledge of Jewish thought, the author has also studied the problems relating to bio-ethics as well as having analysed the existence of various fields of knowledge, thus confronting different models of rationality in their quest for truth (A tort et à raison. Seuil. 1986). In this article, he analyzes the polysemous nature and the philosophical implications of the concept of chance based on the translation of two verses in the hook of Leviticus, thus underscoring the hermeneutic virtues of translation, which are similar to those of scriptural commentary. 


\title{
COMMENT LE DIEU BIBLIQUE PEUT «ALLER AU HASARD» EN HÉBREU MAIS PAS EN TRADUCTION
}

HeNRI ATLAN

Hoputal Universitare Hadassah, Jérusalem. Isruël

\begin{abstract}
Medical doctor, biologist and professor in Paris and Jerusalem, the author has devel. oped theories on the complexity and the self-organization of living organisms (Entre le cristal et la fumee, Seuil. 1979). Drawing on his profound knowledge' of Jew'ish thought, the author has also studied the problems relating to bio-ethics as well as having analysed the existence of various fields of knowledge, thus confronting different models of rationality in their quest for truth (Â tort et à raison. Seuil, 1986). In this article, he analyzes the polysemous nature and the philosophical implications of the concept of chance based on the translation of two verses in the book of Leviticus, thus underscoring the hermeneutic virtues of translation. which are similar to those of scriptural commentary
\end{abstract}

Au cours d'un travail théorique sur le rôle du hasard, sous forme de perturbations aléatoires d'origine externe ou interne dans les mécanismes d'auto-organisation de la matière, je rencontrai dans mes lectures deux versets du chapitre 26 du Lévitique qu'il me semblait intéressant de citer en exergue d'un article, puis d'un livre, sur ce sujet. Dans ces versets, Dieu parle et dit : "Parce qu ils se seront conduits avec moi au hasard, je me conduirai moi aussi avec eux au hasard" (Lévitique 26,40-41). J'avais lu ces versets dans le texte hébreu de la Bible et je les citai en les traduisant moi-même sans me soucier de me référer à telle ou telle traduction, officielle ou autre.

C'est alors que des lecteurs - bien intentionnés - eurent l'idée d'aller vérifier la citation et que deux surprises nous attendaient la, les lecteurs et moi. Première surprise. aucune traduction de la Bible ne rendait, comme je l'avais fait spontanément, le mot «kéri» par «hasard». Deuxième surprise : les hébraïsants parmi mes lecteurs étaient bien d'accord sur l'exactitude de ma traduction littérale concernant ce mot mais ne pouvaient pas l'accepter dans ce contexte, où il s'agit de la conduite des hommes et surtout de celle de Dieu. L'idée que Dieu puisse dire qu'il se comporterait "au hasard" leur semblait proprement incompréhensible, et cela justifiait à leurs yeux que toutes les traductions de la Bible aient été forcées de recourir à des approximations plus ou moins heureuses. Les plus mauvaises des traductions utilisent deux expressions différentes pour le même "bekéri" hébreu, suivant qu'il se rapporte aux hommes ou à Dieu.

Les moins mauvaises des traductions arrivent à trouver une même expression (en français, en anglais, en allemand), mais ce n'est jamais uau hasard». Par exemple. la Bible du rabbinat français, ainsi que $A$. Chouraqui dans sa traduction, rendent le «bekéri» hébreu par «avec hostilité». La traduction de Dhorme (La Pléiade), de son côté, est très habile par son caractère suggestif : «...ils ont marché à l'encontre de moi : ...moi aussi, j'avais marché à l'encontre d'eux" (verset 40-41). "Marcher à l'encontre" peut indiquer à la fois la rencontre d'un événement que suggère la racine "mikreh" de "kéri", et l'antagonisme ou l'hostilité qui caractérise le contexte. Et l'expression du verset 27 dans le même chapitre ( «hamat-kéri", "fureur de hasard" est alors naturellement traduite : "...c'est avec fureur que je marcherai à l'encontre de vous et que je vous corrigerai moi-même au septuple de vos péchés". 
Ce qui rend les choses encore plus compliquées est que ce mot «kéri» n'est employé dans la Bible que dans ce contexte, dans ce seul chapitre du Lévitique, aux versets déjà cités, ainsi qu'aux versets 21 : «Et si vous allez avec moi au hasard...»; 23 et $24:$ «... et si vous allez avec moi au hasard, $\mathrm{j}$ 'irai moi aussi avec vous au hasard...»; 27 et 28 : "Et si en cela vous ne m'écoutez pas et allez avec moi au hasard, j'irai avec vous avec une colère (ou un échauffement) de hasard (behamat-kéri)». II s'agit toujours, dans ce chapitre, de l'annonce des malédictions qui s'abattront sur le peuple d'Israël quand il abandonnera l'alliance et la loi de son dieu.

Ce n'est que plus tardivement, dans l'hébreu de la Michna (Berakhot III,3), que ce mot «kéri» réapparaît cette fois avec le sens d'émission nocturne de sperme, se produisant accidentellement, «au hasard» au sens de non intentionnellement. La signification de «kéri» est alors celle d'un «mikreh laila», «accident noctume» (Deutéronome 23,11), où le mot mikreh de la racine karoh, beaucoup plus utilisé, a le sens bien connu d'événement survenant "au hasard" au sens d'accidentellement, de façon irrégulière et non intentionnelle.

Je traduisais donc ces versets tels que je les comprenais dans ce qui m'apparaissait spontanément et immédiatement comme leur signification littérale. Cette signification est en gros la suivante :

L'existence ou non d'un sens des événements naturels dépend du comportement des hommes par rapport à la nature et de leur faculté de domestiquer le hasard, c'est-à-dire de lui donner un sens. Si les hommes vont au hasard dans la nature, c'est-à-dire considèrent qu'il n'y a aucun sens, alors en effet il n'y a aucun sens et les événements s'y produisent en effet au hasard, "Dieu va au hasard avec eux». Visiblement, cette conception du comportement de Dieu déterminé par celui des hommes, suivant le principe talmudique de "mida keneged mida", "mesure contre mesure», et largement développée par la littérature du midrash et de la kabbale. heurte des conceptions théologiques bien enracinées au point que cela en devient incompréhensible et inimaginable. N'ayant, quant à moi, aucun a priori théologique, $\mathrm{j}$ 'avais compris et traduit spontanément ce texte dans sa signification littérale et y avais trouvé d'ailleurs un certain accent nietzschéen au point de lui associer une citation de Zarathoustra :

Je fais bouillir dans ma marmite tout ce qui est hasard. Et ce n'est que lorsque le hasard est cuit à point que je lui souhaite la bienvenue pour en faire ma nourriture. Et en vérité. maint hasard s'est approché de moi en man̂tre: mais ma volonté lui parle d'une façon plus impérieuse encore, - el aussitôt. il se mettait à genoux devant moi en suppliant - me suppliant de lui donner asile et accueil cordial, et me parlant d'une manière flatteuse : «Vois donc, Zarathoustra, il n'y a qu'un ami pour venir ainsi chez un ami!" (Ainsi parlait Zarathoustra, III. 5-3).

Toutefois, surpris après coup par l'originalité bien involontaire dont $\mathrm{j}$ 'avais ainsi fait preuve, je recherchai alors dans les commentaires traditionnels une confirmation au fait que je n'avais pas été seul à lire le texte hébreu de cette façon. Et en effet, le commentaire canonique de Rachi sur le verset 21 mentionne bien le sens du mot "mikreh". événement se produisant accidentellement (bearai), comme la façon la plus commune de comprendre le sens du mot abekéri» dans ce verset.

Appliquée au comportement humain, cette marche "au hasard" est alors comprise comme une observance non permanente et irrégulière de la Loi, intermittente, «au hasard». Reste alors à comprendre ce que cette expression peut bien vouloir dire quand elle est appliquée au comportement de Dieu. Un autre exégète, un peu moins connu, Rav Hayim Ben Attar, auteur du commentaire Or Hahayim sur la Torah, répond à cette question de la façon suivante à propos des versets 23 et 24 . Les malédictions déclenchées 
par l'abandon de l'alliance se produisent normalement en relation avec les transgressions. Dans un premier temps d'éloignement de la Loi, le mal que produit une transgression est en relation avec cette transgression, et sa signification est évidente comme sanction de cette transgression. Mais lorsque les hommes persistent et s'éloignent encore plus, à tel point que leur relation à la Loi n'est qu'épisodique, accidentelle, «au hasard», alors le mal qui en résulte apparaît lui aussi sans signification, sans relation à quelque transgression que ce soit, frappant lui aussi «au hasard». Pourtant, en dehors de ces indications, je ne trouvai pas tout de suite de confirmation sans équivoque, au moins chez certains lecteurs traditionnels de la Bible en hébreu, de ce que ma traduction rendait la signification évidente de ce texte. Ce n'est que plusieurs années plus tard que je lisais un texte qui n'avait plus été réédité depuis plusieurs siècles et qui, de ce fait, était encore moins connu, le traité kabbaliste Guinat Egoz, «Jardin de noix» (parfois traduit par "Jardin de l'amandier»!) écrit au XIII ${ }^{\mathrm{e}}$ siècle par Rabbi Joseph Guikatilia : «Le mot «kéri» indique une catégorie d'où la providence a disparu, avec le sens d'abandon, (hefker) en ce que les choses arrivent sans intention comme quelque chose qui se produit par la force du hasard (kéri) sans conscience ni intention et sans quelque élément que ce soit de providence ou de réflexion. Et c'est de ce hasard-là (kéri) qu'il s'agit dans la Torah (Lévitique 26, 21) comme pour dire : «Si vous niez ma providence et si vous dites que tous les biens et les maux qui vous arrivent, arrivent par hasard (derekh kéri) sans élément providentiel et sans élément intentionnel de récompense et de châtiment, alors je ferai disparaître ma providence de tout [ce qui vous arrivera] et vous serez complètement abandonnés (hefker) à ce qui se produira ( «se fera-être») à partir d'une "catégorie de hasard" (ythaveh me'inian kéri). C'est cela qu'exprime le verset 24 : 'J'irai moi aussi avec vous au hasard'. C'est cela, la disparition totale de sa providence - qu'il soit béni». (J. Guikatilia, Guinat Egoz, p. 326, Édition Yechiva hahayim vehachalom, Jérusalem, 1989). Le texte de J. Guikatilia vaut la peine d'être évoqué en détails. Il s'attache en fait à montrer la différence de sens entre kéri et mikreh malgré leur racine commune. Ce n'est pas sans importance car cela explique l'usage inusité du mot akérì dans ce chapitre qui ne se justifierait pas s'il avait le même sens que mikreh. Pour l'auteur de Guinat Egoz, la différence tient précisément en ce que seul kéri désigne l'événement aléatoire, "hefker". c'est-à-dire littéralement «abandonné» au sens de «n'appartenant à personne», «sous la souveraineté de personne». ne renvoyant a aucune intention. "Mikrch», au contraire, désignerait la rencontre d'un événement éventuellement porteur de signification quand il est le produit d'une action intentionnelle (d'une «kavanah»). C'est ainsi très explicitement que dans ce chapitre la conduite des Hébreux et celle de leur Dieu est présentée comme sans signification, conduite aléatoire et désordonnée d'hommes sans loi et conduite de Dieu, "c'est-à-dire la nature», sans aucun sens par rapport aux hommes, sans "providence». L'expression "hamar kéri", «fureur de hasard", nous suggère alors naturellement l'image du «bruit et de la fureur» d'une histoire absurde et violente où se débattent les hommes ainsi abandonnés. Mais le commentaire de J. Guikatilia contient un élément de plus, tout à fait remarquable par l'éclairage qu'il apporte sur les rapports entre "hasard" et loi naturelle, entre signification éventuelle de l'existence humaine et déterminismes naturels. Dans les versets 21 et 27 de notre chapitre du Lévitique, l'expression “j'irai au hasard avec vous» est suivie de la menace de frapper les Hébreux «au septuple de leurs fautes". Ce "septuple" est interprété par (iuikatilia comme une allusion aux sept planètes qui désignent pour lui, dans le contexte astrologique de la science de l'Antiquité et du Moyen Âge, les principaux canaux par où s'exercent les déterminismes naturels. 11 est remarquable que la marche du dieu d'Israël par rapport à son peuple, "au hasard" des événements quand ceux-ci sont eux-mêmes produits au hasard des compontements humains, coexiste avec l'ordre rigide du déterminisme de la nature. Le hasard en question 
n'est donc pas absence d'ordre. Il n'est qu'absence de sens par rapport à l'ordre des significations humaines, c'est-à-dire intentionnelles et éventuellement morales. Est-ce à dire, inversement, que les comportements divins «providentiels», c'est-à-dire la survenue d'événements porteurs de sens dans l'histoire des hommes, individuelle ou collective, échapperaient aux déterminismes naturels? L'expression talmudique «Ein mazal le Israel" ("Il n'y a pas de déterminisme astral pour Israèl») pourrait le laisser supposer : mais elle est contredite dans le Talmud lui-même ; elle demande donc à être interprétée dans le contexte strictement moral ou elle est utilisée (Talmud de Babylone, Chabbat : 156a). L'interprétation que suggère ce contexte rejoint alors l'expression «Tout est entre les mains des cieux [c'est-à-dire déterminé par les astres] sauf la crainte des cieux» (Talmud de Babylone, Berakhot : 33b) énoncée par Rabbi Hanina, le tenant, par ailleurs, de l'opinion contradictoire «ll y a un déterminisme astral pour Israèl»l. Quoi qu'il en soit, R. Joseph Guikatilia développe dans son ouvrage une ontologie qui rejette cette hypothèse où un monde du sens échapperait aux déterminismes naturels. Ceux-ci, au contraire, reçoivent leur "vérité" et leur intelligibilité de structures formelles où s'expriment les «essences» éternelles des choses existantes. Ces essences dérivent toutes du «nom de l'essence» (le tétragramme) par le biais de combinaisons, de permutations et de multiplications des lettres qui le constituent. Pour décrire le rapport de ces essences a la nature, il reprend à son compte les expressions scolastiques de "nature naturée» et d'action «naturante» en les hébraïsant et en les rapportant à différents noms de Dieu2. Ainsi, comme je l'avais spontanément imaginé à la lecture du Lévitique en hébreu, le rôle du kéri comme hasard dans la nature répondant au hasard des comportements humains s'inscrit sans aucune difficulté dans une ontologie où la nature des comportements de Dieu (c'est-àdire de la Nature) est intelligible et porteuse de sens ou absurde, suivant les représentations projectives que s'en font les hommes et les comportements qui s'ensuivent.

À ma connaissance, ce texte de J. Guikatilia dans Guinat Egoz, avec le commentaire de Or Hahayim cité plus haut (lui aussi d'inspiration kabbaliste), est le seul témoignage ${ }^{3}$ de ce que le mot "kéri» dans ce chapitre du Lévitique fut compris par des lecteurs hébraisants avec son sens de hasard, même dans son application au comportement de Dieu.

Cette histoire de traduction nous apprend plusieurs choses : d'une part. l'influence parfois gênante de préjugés théologiques dans la compréhension littérale de la Bible : d'autre part, la liberté de certains rabbins kabbalistes par rapport à ces préjugés, résultant probablement de ce que la tradition kabbaliste est profondément plus cosmologique que théologique. L'objet de la connaissance y est beaucoup plus le monde que Dieu. même si celui-là peut y être vu comme activité de celui-ci. Enfin, la discussion talmudique sur le sens ou le non-sens des souffrances de la maladie (Talmud de Babylone, Berakhot : 5a) acquiert un éclairage intéressant dans le cadre de cette conception «mécanique» d'un sens qui n'existerait objectivement que dans l'exacte mesure où les hommes le font exister. Dans cette discussion, les souffrances infligées par la nature sont interprétées soit comme signes de fautes à rechercher, soit, «après avoir cherché et n'avoir pas trouvé»", comme «épreuve d'amour». Celles-ci, on peut alors soit les accepter comme telles dans une ascèse où l'épreuve surmontée est censée fortifier et apparaît en fin de compte comme bénéfique : soit au contraire les rejeter «elles et leurs récompenses» (ihid. : 5b) - c'est-àdire décliner les bénéfices qu'elles seraient susceptibles de produire ultérieurement, dans le monde «à venir». Dans cette demière éventualité, on ne peut que les rapporter à l'œuvre «naturelle» de parasites et de «démons» maléfiques qu'on doit combattre et dont il faut se débarrasser.

Nous sommes ainsi conduits à l'image d'une superposition possible de deux déterminismes, celui de la Nature, sans signification humaine, et celui d'une intelligibilité spé- 
cifiquement humaine, essentiellement morale. C'est cette intelligibilité-là que Job se lamente de ne pas percevoir dans ses malheurs. Mais l'introduction et la conclusion du livre de Job montrent que le tort de Job et de ses amis était de chercher le sens de ces malheurs dans l'intelligibilité simpliste d'une morale infantile, où les vertus seraient automatiquement récompensées par des bienfaits de la nature, et les crimes punis par des catastrophes tout aussi naturelles. Plus exactement, c'est le même déterminisme qui est absurde ou intentionnel suivant le monde de significations, impersonnelles ou personnelles, respectivement, que fabriquent les hommes comme êtres de discours et de pensées, quand ils sont aux prises avec le réseau de leurs propres actions et des événements naturels qui les traversent.

Certains se demanderont si c'est vraiment cela que "voulait dire" le texte original. Mais cette question a-t-elle un sens? Si ce que veut dire un texte doit être dit par un autre texte, il est probable que celui-ci dira autre chose. La traduction, de ce point de vue, peut ajouter au texte et pas seulement retrancher. Quand le texte original est vieux de plusieurs millénaires, toute lecture, même dans la langue d'origine. fonctionne nécessairement comme une traduction. Et c'est en quoi les traductions explicites de ces textes sont indispensables à leur compréhension, même si chacune d'elles en donne une image partielle. C'est leur réunion dans un ensemble, potentiellement infini, qui permet de reconstruire une signification qui, d'une certaine façon, leur appartient. Car peut-on concevoir raisonnablement que les mêmes mots "veuillent dire" la même chose à des milliers d'années d'intervalle ? Le mot «nature» (teva) n'existe pas en hébreu biblique. Il n'apparaît que dans l'hébreu médiéval à partir de la racine du verbe biblique tava «s'enfoncer". par le relais probable de l'hébreu de la Michna (Sanhedrin 4,5) où le même verbe est utilisé avec le sens de graver, imprimer une forme (nature?) humaine à l'aide d'un «sceauanneau» (taba'at, toujours la même racine). À plus forte raison peut-on douter de la pertinence de la notion de hasard dans la nature appliquée au texte biblique. au sens moderne d'aléatoire, ou de non intentionnel, ou encore plus, de «rencontre de deux séries causales indépendantes" suivant la définition classique de Cournot. Mais il en est de même de toutes les notions, y compris et surtout de celle de Dieu, chargée par les siècles d'une théologie qu'ont véhiculée le grec, le latin et l'arabe. Notons que la Septante traduit kéri dans nos versets du Lévitique par plagios, «oblique», «de travers»; et non par kairos ni automatein, deux expressions pour «hasard", mais bien éloignées elles aussi du sens moderne, naturaliste, d'événement aléatoire. Pourtant, peut-être plagios est-il plus proche d'une notion ancienne de kéri où le hasard serait le désordonné, qui survient "de travers" par rapport à l'ordre de la pensée droite. Mais la raison rusée, la métis, permettrait de l'utiliser, tout comme elle est capable de retourner l'inattendu à son profit's. Ruse contre ruse. Nous retrouverions alors par ce détour le mida kenegued mida du kéri contre kéri. Le grec ancien, de par sa proximité historique avec l'hébreu de la Bible et de la Michna. en serait-il sémantiquement plus proche que les langues véhiculaires de la théologie classique, au moins en ce qui concerne notre histoire de "hasard" ?

Peut-être, mais les traductions araméennes de ces mêmes versets nous montrent que ce n'esı pas aussi simple. L'araméen est probablement la langue la plus proche de l'hébreu biblique, dans le temps et dans sa structure. Et pourtant, deux traductions, l'une de Jonathan ben Ouziel, l'autre de Onkelos, traduisent kéri de façon différente. La première rend bien hekéri par he' arai (comme l'indique Rachi), c'est-à-dire «par accident". Mais si elle utilise le même mot be'arai pour Dieu et pour les hommes, elle utilise pourtant deux expressions verbales différentes : «aller par accident (avec Dieu)» pour les hommes, est changé en "se conduire par accident de façon commune» (ou "simplement», he' alma, littéralement "par le monde»), quand il s'agit de Dieu. C'est ainsi que les versets 23 et 24. par exemple, sont traduits par : «...si vous allez avec moi par accident, moi 
je me conduirai simplement avec vous par accident...". Comme si, malgré tout, la symétrie du texte hébreu était difficile à conserver. Quant à la deuxième traduction, celle d'Onkelos, elle traduit bekéri dans tous les cas par bekachiou, c'est-à-dire «avec dureté. résistance, opposition», comme dans le "peuple à la nuque raide» littéralement kchei 'oref «durs de nuque». Ici. c'est l'élément d'opposition qui est retenu dans la rencontre avec l'événement (mikreh) qui survient sans qu'on l'ait appelé, que nous rencontrons devant nous, qui nous heurte. On retrouve là le «a l'encontre de» de La Pléiade. Les deux aspects d'imprévu et d'opposition réunis dans kéri se trouvent ainsi dissociés dans ces deux traductions araméennes. Mais c'est cela même qui permet de les mettre à jour, et là se trouve l'intérêt de traduire.

Notes

1. Voir, sur cette discussion, H. Atlan. À tort et a raison. Intercritique de la Science el du Mythe, Paris, Seuil, 1986, pp. 247-248.

2. On sait l'usage que fera plus tard Spinoza de ces expressions en les incorporant à sa philosophie naturelle. Par rappon aux theologies de la transcendance d'inspiration aristotelicienne, tout se passe comme si celui-ci avait pousst a l'extreme un processus de naturalisation de la notion d'essence dans le cadre d'une pensée de l'immanence. Ce processus, qui rappelle la théologie stö̈cienne et aussi quelques aspects du néoplatonisme syncrétiste de la Renaissance. semble être amorce chez J. Guikatilia.

3. Comme nous l'avons indique. Rachi, elliptique comme à son habitude, le comprend aussi de cette facon (tout en citant d'autres possibilites) à propos du comportement des hommes, et laisse le soin au lecteur de s'interroger sur ce qui en resulte quant au comportement de Dieu.

4. Je suis redevable a Irun $\mathbf{R}$. Cohen d'avoir attire mon attention sur la signification du commentaire de Rachi à cet endroit.

5. Voir M. Detienne et J. P. Vernant, Les ruses de l'intelligence. La Metis des Grecs, Paris, Flammarion, 1974 : et H. Atlan, A tort et d rasson, op.cis., chap. 3. section 7. 